\title{
Some Generalized Information Inequalities
}

\author{
Ram Naresh Saraswat \\ Department of Mathematics, ManipalUniveristy, Jaipur \\ Rajasthan-303007 INDIA,
}

\begin{abstract}
Information inequalities are very useful and play a fundamental role in the literature of Information Theory. Applications of information inequalities have discussed by well-known authors like as Dragomir, Taneja and many researchers etc. In this research paper, we shall consider some new functional information inequalities in the form of generalized information divergence measures. We shall also consider relations between Csiszar's f-divergence, new f-divergence and other well-known divergence measures using information inequalities. Numerical bounds of information divergence measure have also studied.
\end{abstract}

\section{KEYWORD}

Csiszar's f-divergence, New f-divergence measure, Relative information of type's, J-divergence of type's, Relative J-divergence of type'setc.

\section{INTRODUCTION}

Let

$$
\Gamma_{n}=\left\{P=\left(p_{1,} p_{2,} \ldots \ldots . . . p_{n}\right) \mid p_{i} \geq 0, \sum_{i=1}^{n} p_{i}=1\right\}, n \geq 2
$$

be the set of all complete finite discrete probability distributions. There are many information and divergence measures are exist in the literature of Information Theory and Statistics. Csiszar [2] \& [3] introduced a generalized measure of information using f-divergence measure given by

$$
I_{f}(P, Q)=\sum_{i=1}^{n} q_{i} f\left(\frac{p_{i}}{q_{i}}\right)
$$

where $f: \mathbf{R}_{+} \rightarrow \mathbf{R}_{+}$is a convex function and $P, Q \in \Gamma_{n}$.

As in Csiszar [3], we have interpret undefined expressions by Csiszar's f-divergence is a general class of divergence measures that includes several divergences used in measuring the distance or affinity between two probability distributions. This class is introduced by using a convex function $\mathrm{f}$, defined on $(0, \infty)$.

Here we give some examples of divergence measures which are the category of Csiszar's fdivergence measure like as Bhattacharya divergence [1], Triangular discrimination [4], Relative J-divergence [5], Hellinger discrimination [6], Chi-square divergence [9], Relative Jensen- 
Shannon divergence [13], Relative arithmetic-geometric divergence measure [10], Unified relative Jensen-Shannon and arithmetic-geometric divergence measure[10].

Now, we give some examples of well-knowngeneralized information divergence measures of type's which are obtained from Csiszar's f-divergence measure.

- Relative information of type s [11]

The following measures and particular cases are introduced by Taneja [11]

$$
\Phi_{s}(P, Q)=\left\{\begin{array}{lr}
{ }^{2} K_{s}(P, Q)=[s(s-1)]^{-1}\left[\sum_{i=1}^{n} p_{i}^{s} q_{i}^{1-s}-1\right], & s \neq 0,1 \\
D(Q, P)=\sum_{i=1}^{n} q_{i} \log \left(\frac{q_{i}}{p_{i}}\right), & s=0 \\
D(P, Q)=\sum_{i=1}^{n} p_{i} \log \left(\frac{p_{i}}{q_{i}}\right), & s=1
\end{array}\right.
$$

and

$$
\eta_{s}(P, Q)= \begin{cases}(s-1)^{-1} \sum_{i=1}^{n}\left(p_{i}-q_{i}\right)\left(\frac{p_{i}}{q_{i}}\right)^{s-1}, & s \neq 1 \\ J(P, Q)=\sum_{i=1}^{n}\left(p_{i}-q_{i}\right) \log \left(\frac{p_{i}}{q_{i}}\right), & s=1\end{cases}
$$

- Relative J-divergence of type s [12]

$$
\varsigma_{s}(P, Q)= \begin{cases}D_{s}(P, Q)=[s-1]^{-1} \sum_{i=1}^{n}\left(p_{i}-q_{i}\right)\left(\frac{p_{i}+q_{i}}{2 q_{i}}\right)^{s-1}, & s \neq 1 \\ J(P, Q)=\sum_{i=1}^{n} p_{i} \log \left(\frac{p_{i}}{q_{i}}\right), & s=1\end{cases}
$$

\section{NEW F-DIVERGENCE MEASURE}

In this section we shall consider some properties of a new f-divergence measure [Jain and Saraswat [7] \& [8] and its particular cases which are may be interesting in areas of information theory is given by

$$
S_{f}(P, Q)=\sum_{i=1}^{n} q_{i} f\left(\frac{p_{i}+q_{i}}{2 q_{i}}\right)
$$

Where $f: \mathbf{R}_{+} \rightarrow \mathbf{R}_{+}$is a convex functionand $P, Q \in \Gamma_{n}$.

It is shown that using new f-divergence measure we derive some well-known divergence measures such as Chi-square divergence, Relative J-divergence, Jenson-Shannon's divergence, Triangular discrimination, Hellinger discrimination, Bhattacharya divergence, Unified relative Jensen-Shannon and arithmetic-geometric divergence of type'setc. in this section. 
The following propositions are presented by Jain \&Saraswat in [7] \& [8].

Proposition 2.1 Let $f:[0, \infty) \rightarrow \mathbf{R}$ be convex and $P, Q \in \Gamma_{n}$ with $P_{n}=Q_{n}=1$ then we have the following inequality

$$
S_{f}(P, Q) \geq f(1)
$$

Equality holds in (2.2) iff

$$
p_{i}=q_{i} \forall i=1,2, . ., n
$$

Corollary 2.1.1 (Non-negativity of new f-divergence measure) Let $f:[0, \infty) \rightarrow \mathbf{R}$ be convex and normalized, i.e.

$$
f(1)=0
$$

Then for any $P, Q \in \Gamma_{n}$ from (2.2) of proposition 2.1 and (2.4), we have the inequality

$$
S_{f}(P, Q) \geq 0
$$

If $f$ is strictly convex, equality holds in (2.5) iff

$$
p_{i}=q_{i} \quad \forall i \in[i, 2, \ldots \ldots \ldots \ldots \ldots n]
$$

In particular, if $\mathrm{P} \& \mathrm{Q}$ are probability vectors, then Corollary 2.1.1 shows that for a strictly convex and normalized $f:[0, \infty) \rightarrow \mathbf{R}$

$$
S_{f}(P, Q) \geq 0 \text { and } S_{f}(P, Q)=0 \text { iff } P=Q
$$

Proposition 2.2 Let $f_{1} \& f_{2}$ are two convex functions and $g=a f_{1}+b f_{2}$ then $S_{g}(P, Q)=a S_{f_{1}}(P, Q)+b S_{f_{2}}(P, Q)$, where $P, Q \in \Gamma_{n}$.

We now give some examples of well-known information divergence measures which are obtained from new f-divergence measure.

- Chi-square divergence measure: - If $f(t)=(t-1)^{2}$ then Chi-square divergence measure is given by

$$
S_{f}(P, Q)=\frac{1}{4}\left[\sum_{i=1}^{n} \frac{p_{i}^{2}}{q_{i}}-1\right]=\frac{1}{4} \chi^{2}(P, Q)
$$

- Relative Jensen-Shannon divergence measure:-If $f(t)=-\log t$ then relative Jensen-Shannon divergence measure is given by 


$$
S_{f}(P, Q)=\sum_{i=1}^{n} q_{i} \log \left(\frac{2 q_{i}}{p_{i}+q_{i}}\right)=F(Q, P)
$$

- Relative arithmetic-geometric divergence measure:-If $f(t)=t \log t$ thenrelative arithmeticgeometric divergence measure is given by

$$
S_{f}(P, Q)=\sum_{i=1}^{n}\left(\frac{p_{i}+q_{i}}{2}\right) \log \left(\frac{p_{i}+q_{i}}{2 q_{i}}\right)=G(Q, P)
$$

- Triangular discrimination: - If $f(t)=\frac{(t-1)^{2}}{t}, \forall t>0$ then Triangular discrimination is given by

$$
S_{f}(P, Q)=\sum_{i=1}^{n} \frac{\left(p_{i}-q_{i}\right)^{2}}{2\left(p_{i}+q_{i}\right)}=\frac{1}{2} \Delta(P, Q)
$$

- Relative J-divergence measure: - If $f(t)=(t-1) \log t$ then Relative J-divergence measure is given by

$$
S_{f}(P, Q)=\sum_{i=1}^{n}\left(\frac{p_{i}-q_{i}}{2}\right) \log \left(\frac{p_{i}+q_{i}}{2 q_{i}}\right)=\frac{1}{2} J_{R}(P, Q)
$$

- Hellinger discrimination: - If $f(t)=(1-\sqrt{t})$ then Hellinger discrimination is given by

$$
S_{f}(P, Q)=\left[1-B\left(\frac{P+Q}{2}, Q\right)\right]=h\left(\frac{P+Q}{2}, Q\right)
$$

\section{INFORMATION INEQUALITIES}

The following Theorems 3.1 presented in Taneja\&Pranesh Kumar [11] and Theorem 3.2 presented in Jain\&Saraswat [7] respectively.

Theorem 3.1Let $f: \mathbf{R}_{+} \rightarrow \mathbf{R}$ be the differentiable convex function and normalized i.e. $f(1)=0$. Then for all $P, Q \in \Gamma_{n}$ we have the following inequality

where

$$
\begin{aligned}
& 0 \leq I_{f}(P, Q) \leq W_{I_{f}}(P, Q) \\
& W_{I_{f}}(P, Q)=\sum_{i=1}^{n}\left(p_{i}-q_{i}\right) f^{\prime}\left(\frac{p_{i}}{q_{i}}\right)
\end{aligned}
$$

In addition, if we have $0<r \leq \frac{p_{i}}{q_{i}} \leq R<\infty, \forall i \in\{1,2, \ldots \ldots \ldots \ldots . . ., n\}$ for some $\mathrm{r}$ and $\mathrm{R}$ with $0<r \leq 1 \leq R<\infty$, then the followings holds 


$$
\begin{aligned}
& I_{f}(P, Q) \leq W_{I_{f}}(P, Q) \leq \frac{1}{4}(R-r)\left[f^{\prime}(R)-f^{\prime}(r)\right] \\
& I_{f}(P, Q) \leq \frac{(R-1) f(r)+(1-r) f(R)}{R-r} \leq \frac{1}{4}(R-r)\left[f^{\prime}(R)-f^{\prime}(r)\right]
\end{aligned}
$$

Theorem 3.2:-Let $f: I \subset \mathbf{R}_{+} \rightarrow \mathbf{R}_{+}$be a differentiable convex mapping on $\stackrel{0}{I}$. If $x_{i} \in \stackrel{0}{I}$ and . $P, Q \in \Gamma_{n}$. Then we have the following inequality,

$$
0 \leq S_{f}(P, Q)-f(1) \leq \frac{1}{2} \sum_{i=1}^{n}\left(p_{i}-q_{i}\right) f^{\prime}\left(\frac{p_{i}+q_{i}}{2 q_{i}}\right)
$$

where

$$
S_{f}(P, Q)=\sum_{i=1}^{n} q_{i} f\left(\frac{p_{i}+q_{i}}{2 q_{i}}\right)
$$

and $f^{\prime}$ is derivative of $f$. If $f$ is strictly convex, and $p_{i}, q_{i}>0,(i=1,2, \ldots \ldots . . n)$ then the equality holds in (3.4) iff $\frac{p_{1}}{q_{1}}=\frac{p_{2}}{q_{2}}=\ldots \ldots \ldots \ldots \ldots . . . . \frac{p_{n}}{q_{n}}$.

Corollary 3.1If function $f$ is normalized i.e. $f(1)=0$ then we have the following inequality

where

$$
\begin{gathered}
0 \leq S_{f}(P, Q) \leq \sum_{i=1}^{n}\left(\frac{p_{i}-q_{i}}{2}\right) f^{\prime}\left(\frac{p_{i}+q_{i}}{2 q_{i}}\right) \\
0 \leq S_{f}(P, Q) \leq E_{S_{f^{\prime}}}(P, Q) \\
E_{S_{f^{\prime}}}(P, Q)=\sum_{i=1}^{n}\left(\frac{p_{i}-q_{i}}{2}\right) f^{\prime}\left(\frac{p_{i}+q_{i}}{2 q_{i}}\right)
\end{gathered}
$$

\section{NEW FUNCTIONAL INFORMATION INEQUALITIES}

In this section we shall consider some new functionalinformation inequalities among various $\mathrm{f}$ divergences. Using these functional information inequalities, we shall establish relations between well-known generalized information divergence measures and its particular cases.

Theorem 4.1Let $f: I \subset \mathbf{R}_{+} \rightarrow \mathbf{R}_{+}$be the differentiable convex function and normalized i.e. $f(1)=0$. Then for all $P, Q \in \Gamma_{n}$ we have the following inequality

$$
\begin{aligned}
& S_{f}(P, Q) \leq E_{S_{f^{\prime}}}(P, Q) \leq I_{f}(P, Q) \leq W_{I_{f}}(P, Q) \leq \frac{1}{4}(R-r)\left[f^{\prime}(R)-f^{\prime}(r)\right] \\
& S_{f}(P, Q) \leq E_{S_{f^{\prime}}}(P, Q) \leq I_{f}(P, Q) \leq \frac{(R-1) f(r)+(1-r) f(R)}{R-r} \\
& S_{f}(P, Q) \leq E_{S_{f^{\prime}}}(P, Q) \leq I_{f}(P, Q) \leq \frac{(R-1) f(r)+(1-r) f(R)}{R-r} \leq \frac{1}{4}(R-r)\left[f^{\prime}(R)-f^{\prime}(r)\right]
\end{aligned}
$$


Proof: - As $f$ is differential convex on $I$, then for all $x, y \in \stackrel{0}{I}$, we have the inequality

$$
(x-y) f^{\prime}(y) \leq f(x)-f(y), \forall x, y \in \stackrel{0}{I}
$$

Now we take $y=\frac{x+1}{2}$

$\left(x-\frac{x+1}{2}\right) f^{\prime}\left(\frac{x+1}{2}\right) \leq f(x)-f\left(\frac{x+1}{2}\right)$

$\left(\frac{x-1}{2}\right) f^{\prime}\left(\frac{x+1}{2}\right) \leq f(x)-f\left(\frac{x+1}{2}\right)$

Put $x=\frac{p_{i}}{q_{i}}$

$\left(\frac{p_{i}-q_{i}}{2 q_{i}}\right) f^{\prime}\left(\frac{p_{i}+q_{i}}{2 q_{i}}\right) \leq f\left(\frac{p_{i}}{q_{i}}\right)-f\left(\frac{p_{i}+q_{i}}{2 q_{i}}\right)$

Multiplying by $q_{i}$ and taking summation both side then we get

$$
\begin{aligned}
& \sum_{i=1}^{n}\left(\frac{p_{i}-q_{i}}{2}\right) f^{\prime}\left(\frac{p_{i}+q_{i}}{2 q_{i}}\right) \leq \sum_{i=1}^{n} q_{i} f\left(\frac{p_{i}}{q_{i}}\right)-\sum_{i=1}^{n} q_{i} f\left(\frac{p_{i}+q_{i}}{2 q_{i}}\right) \\
& E_{S_{f^{\prime}}}(P, Q) \leq I_{f}(P, Q)-S_{f}(P, Q) \\
& E_{S_{f^{\prime}}}(P, Q)+S_{f}(P, Q) \leq I_{f}(P, Q)
\end{aligned}
$$

Hence we get

$$
E_{S_{f}}(P, Q) \leq I_{f}(P, Q)
$$

From (3.5)\& (4.5), we get

$$
S_{f}(P, Q) \leq E_{S_{f^{\prime}}}(P, Q) \leq I_{f}(P, Q)
$$

Form (3.1) \& (4.7), we get

$$
S_{f}(P, Q) \leq E_{S_{f^{\prime}}}(P, Q) \leq I_{f}(P, Q) \leq W_{I_{f}}(P, Q)
$$

From (3.2) \& (4.7) give the result (4.1)and (3.3) \& (4.8) give the results (4.2) \& (4.3) respectively.

\section{APPLICATIONS IN INFORMATION THEORY}


In this section we shall establish relationship between various known generalized information measures of type's in the form of inequality using the results (4.3), (4.4) and (4.5) of Theorem (4.1).In the following Theorem we shall consider the applications of functional information inequalities for relations between Unified relative Jensen-Shannon and arithmetic-geometric divergence of type's, Relative J-divergence of type's, Relative information of type's etc.

Theorem: 5.1- Let $P, Q \in \Gamma_{n}$, then we have the following relations

$$
\begin{aligned}
& \Omega_{s}(Q, P) \leq \frac{1}{2} \varsigma_{s}(P, Q) \leq \Phi_{s}(P, Q) \leq \eta_{s}(P, Q) \leq U_{s}^{(r, R)} \\
& \Omega_{s}(Q, P) \leq \frac{1}{2} \varsigma_{s}(P, Q) \leq \Phi_{s}(P, Q) \leq T_{s}^{(r, R)} \\
& \Omega_{s}(Q, P) \leq \frac{1}{2} \varsigma_{s}(P, Q) \leq \Phi_{s}(P, Q) \leq T_{s}^{(r, R)} \leq U_{s}^{(r, R)}
\end{aligned}
$$

where

$$
U_{s}^{(r, R)}=\frac{1}{4} \frac{(R-r)}{(s-1)}\left[R^{s-1}-r^{s-1}\right], T_{s}^{(r, R)}=[s(s-1)]^{-1} \frac{(R-1)\left(r^{s}-1\right)+(1-r)\left(R^{s}-1\right)}{R-r}
$$

Proof: - Considering the mapping $f:(0, \infty) \rightarrow \mathbf{R}$

$$
\begin{aligned}
& f_{s}(t)=[s(s-1)]^{-1}\left(t^{s}-1\right) \quad \text { if } s \neq 0,1 \\
& f^{\prime}(t)=[s-1]^{-1} t^{s-1}, \quad f^{\prime \prime}(t)=t^{s-2} \\
& f^{\prime \prime}(t) \geq 0, \forall t>0 \text { and } f(1)=0, \text { So function } f \text { is convex and normalized. }
\end{aligned}
$$

Then

$$
\begin{aligned}
& S_{f}(P, Q)=\Omega_{s}(Q, P) \\
& E_{S_{f^{\prime}}}(P, Q)=\frac{1}{2} \varsigma_{s}(P, Q) \\
& I_{f}(P, Q)=\Phi_{s}(P, Q) \\
& W_{I_{f}}(P, Q)=\eta_{s}(P, Q) \\
& f_{s}(R)=[s(s-1)]^{-1}\left(R^{s}-1\right) \text { if } s \neq 0,1 \\
& f_{s}(r)=[s(s-1)]^{-1}\left(r^{s}-1\right) \text { if } s \neq 0,1
\end{aligned}
$$

From equation (5.4), (5.9) \& (5.10), we get

$$
T_{s}^{(r, R)}=\frac{(R-1) f(r)+(1-r) f(R)}{R-r}=[s(s-1)]^{-1} \frac{(R-1)\left(r^{s}-1\right)+(1-r)\left(R^{s}-1\right)}{R-r}(5.11)
$$


$U_{s}^{(r, R)}=(R-r)\left[f^{\prime}(R)-f^{\prime}(r)\right]=\frac{1}{4} \frac{(R-r)}{(s-1)}\left[R^{s-1}-r^{s-1}\right]$

Using equation (4.1), (4.2), (4.3) (4.4), (5.4),(5.5),(5.6),(5.7),(5.8),(5.9),(5.10) (5.11) \& (5.12) give the relation (5.1), (5.2) \& (5.3).

In the following corollaries, we shall discuses some cases for particular values of $s=-1,0$, $\frac{1}{2}, 1$ in corollary (5.1), (5.2), (5.3) and (5.4) respectively.

\section{Corollary 5.1:-If $s=-1$}

$$
\begin{aligned}
& \frac{1}{4} \Delta(P, Q) \leq \sum_{i=1}^{n} \frac{p_{i}^{2}\left(p_{i}-q_{i}\right)}{\left(p_{i}+q_{i}\right)^{2}} \leq \frac{1}{2} \chi^{2}(P, Q) \leq \sum_{i=1}^{n}\left(q_{i}-p_{i}\right)\left(\frac{p_{i}}{q_{i}}\right)^{2} \leq \frac{1}{8} \frac{(R-r)^{2}(R+r)}{R^{2} r^{2}} \\
& \frac{1}{4} \Delta(P, Q) \leq \sum_{i=1}^{n} \frac{p_{i}^{2}\left(p_{i}-q_{i}\right)}{\left(p_{i}+q_{i}\right)^{2}} \leq \frac{1}{2} \chi^{2}(P, Q) \leq \frac{1}{2} \frac{r(R-1)(1-r)+(1-r)(1-R)}{r R(R-r)} \\
& \frac{1}{4} \Delta(P, Q) \leq \sum_{i=1}^{n} \frac{p_{i}^{2}\left(p_{i}-q_{i}\right)}{\left(p_{i}+q_{i}\right)^{2}} \leq \frac{1}{2} \chi^{2}(P, Q) \leq \frac{1}{2} \frac{r(R-1)(1-r)+(1-r)(1-R)}{r R(R-r)} \leq \frac{1}{8} \frac{(R-r)^{2}(R+r)}{R^{2} r^{2}}
\end{aligned}
$$

\section{Corollary 5.2If $s=0$}

$$
\begin{aligned}
& F(P, Q) \leq \frac{1}{2} \Delta(P, Q) \leq D(P, Q) \leq \chi^{2}(P, Q) \leq \frac{1}{4} \frac{R-r}{R r}[r \log R-R \log r] \\
& F(P, Q) \leq \frac{1}{2} \Delta(P, Q) \leq D(P, Q) \leq(R-1) \log r-(r-1) \log R \\
& F(P, Q) \leq \frac{1}{2} \Delta(P, Q) \leq D(P, Q) \leq(R-1) \log r-(r-1) \log R \leq \frac{1}{4} \frac{R-r}{R r}[r \log R-R \log r]
\end{aligned}
$$

Corollary 5.3 If $s=1 / 2$

$$
\begin{aligned}
& 4\left[1-B\left(\frac{P+Q}{2}, Q\right)\right] \leq \frac{1}{2} \eta_{\frac{1}{2}}\left(\frac{P+Q}{2}, Q\right) \leq 4 h(P, Q) \leq 2 \sum_{i=1}^{n}\left(q_{i}-p_{i}\right) \sqrt{\frac{q_{i}}{p_{i}}} \leq \frac{1}{2}(r-R)\left[\frac{\sqrt{r}-\sqrt{R}}{\sqrt{R r}}\right] \\
& \text { (5.19) } 4\left[1-B\left(\frac{P+Q}{2}, Q\right)\right] \leq \frac{1}{2} \eta_{\frac{1}{2}}\left(\frac{P+Q}{2}, Q\right) \leq 4 h(P, Q) \leq 4\left[\frac{(1-R)(\sqrt{r}-1)+(1-r)(\sqrt{R}-1)}{R-r}\right]
\end{aligned}
$$

$$
\begin{aligned}
4\left[1-B\left(\frac{P+Q}{2}, Q\right)\right] \leq & \frac{1}{2} \eta_{\frac{1}{2}}\left(\frac{P+Q}{2}, Q\right) \leq 4 h(P, Q) \\
& \leq 4\left[\frac{(1-R)(\sqrt{r}-1)+(1-r)(\sqrt{R}-1)}{R-r}\right] \leq \frac{1}{2}(r-R)\left[\frac{\sqrt{r}-\sqrt{R}}{\sqrt{R r}}\right]
\end{aligned}
$$


Corollary 5.4 For $s=1$

$$
\begin{aligned}
& G(P, Q) \leq \frac{1}{2} J_{R}(P, Q) \leq D(P, Q) \leq J(P, Q) \leq \frac{1}{4}(R-r) \log \left(\frac{R}{r}\right) \\
& G(P, Q) \leq \frac{1}{2} J_{R}(P, Q) \leq D(P, Q) \leq(R-1)[r \log r-R \log R] \\
& G(P, Q) \leq \frac{1}{2} J_{R}(P, Q) \leq D(P, Q) \leq(R-1)[r \log r-R \log R] \leq \frac{1}{4}(R-r) \log \left(\frac{R}{r}\right)
\end{aligned}
$$

\section{NUMERICAL ILLUSTRATIONS}

In this section we shall consider some numerical bounds of f-divergence measure using functional information inequalities and binomial distribution.

\section{Example 6.1}

Let $\mathrm{P}$ be the binomial probability distribution for the random valuable $\mathrm{X}$ with parameter $(\mathrm{n}=8$ $\mathrm{p}=0.5$ ) and $\mathrm{Q}$ its approximated normal probability distribution.

\section{Table 6.1 Binomial Probability Distribution}

$$
(\mathrm{n}=8 \mathrm{p}=0.5)
$$

\begin{tabular}{|c|c|c|c|c|c|c|c|c|c|}
\hline $\mathbf{x}$ & $\mathbf{0}$ & $\mathbf{1}$ & $\mathbf{2}$ & $\mathbf{3}$ & $\mathbf{4}$ & $\mathbf{5}$ & $\mathbf{6}$ & $\mathbf{7}$ & $\mathbf{8}$ \\
\hline $\mathrm{p}(\mathrm{x})$ & .004 & .031 & .109 & .219 & .274 & .219 & .109 & .031 & .004 \\
\hline $\mathrm{q}(\mathrm{x})$ & .005 & .030 & .104 & .220 & .282 & .220 & .104 & .030 & .005 \\
\hline$\frac{p(x)}{q(x)}$ & .774 & 1.042 & 1.0503 & .997 & .968 & .997 & 1.0503 & 1.042 & .774 \\
\hline
\end{tabular}

It is noted that $\quad \mathrm{r}=0.774179933 \approx .77, \mathrm{R}=1.050330018 \approx 1.05$

$$
\begin{aligned}
& T_{s}^{(r, R)}=\frac{(R-1) f(r)+(1-r) f(R)}{R-r}=[s(s-1)]^{-1} \frac{(.05)\left[(.77)^{s}-1\right]+(.33)\left[(1.05)^{s}-1\right]}{(.28)} \\
& T_{s}^{(.77,1.05)}=\frac{5\left[(.77)^{s}-1\right]+33\left[(1.05)^{s}-1\right]}{28[s(s-1)]} \\
& T_{-1}^{(.77,1.05)}=.1533, T_{0}^{(.77,1.05)}=0.01047, T_{1 / 2}^{(.77,1.05)}=.20 \\
& U_{s}^{(.77,1.05)}=\frac{1}{4} \frac{(1.05-.77)}{(s-1)}\left[(1.05)^{s-1}-(.77)^{s-1}\right] \\
& U_{-1}^{(.77,1.05)}=.026819, U_{0}^{(.77,1.05)}=.011, U_{1 / 2}^{(.77,1.05)}=.023, U_{1}^{(.77,1.05)}=.009
\end{aligned}
$$




\section{REFERENCES}

[1]. Bhattacharya A., "Some analogues to amount of information and their uses in statistical estimation", Sankhya8 (1946) 1-14

[2]. Csiszar I. Information measure,A critical servey.Trans. $7^{\text {th }}$ prague conf. on info. Th. Statist. Decius. Funct, Random Processes and $8^{\text {th }}$ European meeting of statist Volume B. Acadmia Prague, 1978, 7386.

[3]. Csiszar I., Information-type measures of difference of probability functions and indirect observations. Studia Sci.Math.hunger.2(1961).299-318.

[4]. Dacunha-Castella D., "Ecoled'Ete de probabilities de Saint-Flour VII-1977 Berline, Heidelberg”, New-York:Springer 1978

[5]. Dragomir S.S., V. Gluscevic and C.E.M. Pearce, "Approximation for the Csiszar f -divergence via mid-point inequalities, in inequality theory and applications- Y. J. Cho, J. K. Kim and S.S. dragomir (Eds), nova science publishers, inc., Huntington, new York, vol1, 2001, 139-154.

[6]. Hellinger "NeueBegrundung der Theorie der quadratischenFormenVonunendlichenvielenveranderlichen”, J.Rein.Aug.Math,136(1909),210-271

[7]. Jain K. C. and R. N. Saraswat "A New Information Inequality and its Application in Establishing Relation among various f-Divergence Measures", Journal of Applied Mathematics, Statistics and Informatics, 8(1) (2012), 17-32.

[8]. Jain K. C. and R. N. Saraswat"Some bounds of information divergence measure in term of Relative arithmetic-geometric divergence measure" International Journal of Applied Mathematics and Statistics, 32,(2) (2013), 48-58.

[9]. Pearson K., "On the criterion that a give system of deviations from the probable in the case of correlated system of variables in such that it can be reasonable supposed to have arisen from random sampling", Phil. Mag., 50(1900),157-172.

[10]. Taneja I.J., "New Developments in generalized information measures", Chapter in: Advances in imaging and Electron Physics, Ed. P. W. Hawkes 91 (1995), 37-135.

[11]. Taneja I. J. and Pranesh Kumar, "Relative Information of type's, Csiszar f-divergence and information inequalities", Information Sciences, 166(1-4) (2004), 105-12

[12]. Taneja I. J. and Pranesh Kumar, "Generalized non-symmetric divergence measures and inequalities".The Natural Science and Engineering Research Council's Discovery grant to Pranesh Kumar

[13]. Sibson R., Information Radius, Z, Wahrs. undverw.geb. (14) (1969), 149-160.

[14]. Wang Yufeng Wang Wendong"A Novel Interest Coverage Method Based On Jensen-Shannon Divergence In Sensor Networks” 24(4) Journal of Electronics (China) July 2007. 